\title{
Induction of Angiogenesis by Matrigel Coating of VEGF-Loaded PEG/PCL-Based Hydrogel Scaffolds for hBMSC Transplantation
}

\author{
Yeon Joo Jung, ${ }^{2,8}$ Kyung-Chul Kim ${ }^{1,8}$, Jun-Young Heo ${ }^{1}$, Kaipeng Jing ${ }^{1,3}$, Kyung Eun Lee ${ }^{2}$, Jun Seok Hwang ${ }^{2}$, \\ Kyu Lim, Deog-Yeon Jo ${ }^{4}$, Jae Pyoung Ahn ${ }^{5}$, Jin-Man Kim ${ }^{6}$, Kang Moo Huh" ${ }^{7, *}$, and Jong-II Park ${ }^{1,3, *}$
}

hBMSCs are multipotent cells that are useful for tissue regeneration to treat degenerative diseases and others for their differentiation ability into chondrocytes, osteoblasts, adipocytes, hepatocytes and neuronal cells. In this study, biodegradable elastic hydrogels consisting of hydrophilic poly(ethylene glycol) (PEG) and hydrophobic poly( $\varepsilon$ caprolactone) (PCL) scaffolds were evaluated for tissue engineering because of its biocompatibility and the ability to control the release of bioactive peptides. The primary cultured cells from human bone marrow are confirmed as hBMSC by immunohistochemical analysis. Mesenchymal stem cell markers (collagen type I, fibronectin, CD54, integrin1 $\beta$, and Hu protein) were shown to be positive, while hematopoietic stem cell markers (CD14 and CD45) were shown to be negative. Three different hydrogel scaffolds with different block compositions (PEG:PCL=6:14 and 14:6 by weight) were fabricated using the salt leaching method. The hBMSCs were expanded, seeded on the scaffolds, and cultured up to 8 days under static conditions in Iscove's Modified Dulbecco's Media (IMDM). The growth of MSCs cultured on the hydrogel with PEG/PCL= 6/14 was faster than that of the others. In addition, the morphology of MSCs seemed to be normal and no cytotoxicity was found. The coating of the vascular endothelial growth factor (VEGF) containing scaffold with Matrigel slowed down the release of VEGF in vitro and promoted the angiogene-

\footnotetext{
${ }^{1}$ Department of Biochemistry, School of Medicine, Chungnam National University, Daejeon 301-747, Korea, ${ }^{2}$ Department of Pharmacology and Medical Research Center, Ewha Womans University School of Medicine, Seoul 158-710, Korea, ${ }^{3}$ Research Institute of Medical School, ${ }^{4}$ Division of Hematology/Oncology Department of Internal Medicine, Chungnam National University, Daejeon 301-747, Korea, ${ }^{5}$ Advanced Analysis Center, Korea Institute of Science and Technology, Seoul 136-791, Korea, ${ }^{6}$ Department of Pathology, School of Medicine, Chungnam National University, Daejeon 301-747, Korea, ${ }^{7}$ Department of Polymer Science and Engineering, Chungnam National University, Daejeon 305-764, Korea, ${ }^{8}$ These authors contributed equally to this work.

*Correspondence: jipark@cnu.ac.kr (JIP); khuh@cnu.ac.kr (KMH)
}

Received 22 May, 2015; revised 10 June, 2015; accepted 12 June, 2015; published online 3 July, 2015

Keywords: angiogenesis, controlled release, hBMSC, hydrogel, VEGF sis when transplanted into BALB/c nude mice. These results suggest that hBMSCs can be supported by a biode gradable hydrogel scaffold for effective cell growth, and enhance the angiogenesis by Matrigel coating.

\section{INTRODUCTION}

The cells that form the human body originate from stem cells; among the different types of adult stem cells, those from the bone marrow are the most extensively studied. Bone-marrow derived mesenchymal stem cells (hBMSCs) that are present in the bone marrow can differentiate into hematopoietic stem cells, osteoblasts, chondrocytes, endothelial cells, and adipocytes (Costa-Pinto et al., 2009; Green et al., 2015; Phinney and Prockop, 2007; Yang et al., 2006). hBMSCs not only support hematopoietic stem cell differentiation but also differentiate into chondrocytes, osteocytes, or adipocytes under appropriate experimental conditions (Pittenger et al., 1999). hBMSCs also promote the bone marrow engraftment of transplanted hematopoietic stem cells. Therefore, hBMSCs have been widely applied in tissue engineering for their multipotency. Cotransplantation of MSCs has been attempted for hematopoietic stem cell transplantation (Zeiser et al., 2004) They have been injected at bone fracture sites as a part of bone regeneration therapy. In addition, MSCs or endothelial cells have been co-cultured for studies in biocompatibility and angiogenesis (Deng, 2010).

Biocompatible and biodegradable scaffolds have been widely investigated in tissue engineering and regenerative medicine to serve as a substrate for cells or a physical support to guide the formation of new extracellular metrix (ECM) (Chen et al., 2015; Mehdizadeh et al., 2013; Peters et al., 2002). Currently, a variety of biomaterial scaffolds, including artificial scaffolds made from hydroxyapatite/tricalcium phosphate or a combination of both (De Kok et al., 2003; Mankani et al., 2001; Tsuchida et al., 2003) and biodegradable polymer scaffolds (Khare and Peppas, 1993; Lee et al., 2001) have been prepared for tissue engineering applications and investigated in terms of biocompatibility and tissue regeneration capacities. Synthetic biodegradable polymer scaffolds may have versatile physico-chemical properties such as mechanical and degradation properties, which can be easily modulated to generate appropriate functional properties in the body. Therefore, many polymer scaffolds have been used as a substrate for tissue regeneration, providing mechani- 
cal and structural support during tissue development (Mehdizadeh et al., 2013).

Ideal scaffolds should be able to fuse with the natural cellular matrix and degrade at a rate commensurate with the elucidation of new ECM by the seeded cells. Thus, the biodegradability of the scaffold is an important criterion for biological tissue and material selection. PCL, one of the representative biodegradable polyesters, has been broadly studied as a biomaterial due to its good biocompatibility. Cell survival significantly increased when PCL was introduced in the body as a thin layer (Hennink et al., 2004). Our previous study showed that poly(ethylene glycol) (PEG) and poly ( $\varepsilon$-caprolactone) (PCL) based hydrogels could be useful for applications and tissue engineering due to several promising properties, such as elasticity, biodegradability, good biocompatibility, and tailor-made physico-chemical properties (Im et al., 2007; Park et al., 2007). In addition, the hydrogels could offer a suitable environment for cell retention and growth.

Recently, biodegradable polymer scaffolds for inducing angiogenesis have attracted much attention as replacement materials for injured or atrophied tissues since they were successfully used to culture stem cells for transplantation (Kanczler et al., 2008; Park et al., 2010; Tanaka et al., 2009; Yang et al., 2010; Zanatta et al., 2012). Angiogenesis, the formation of new capillaries from pre-existing blood vessels, is the primary mechanism for vascularization of tissue engineering (Mehdizadeh et al., 2013). The structure and properties of polymer scaffolds play an important role in regulating tissue growth and angiogenesis. The angiogenesis can be promoted by introducing growth factors such as VEGF, IGF, EGF, PDGF, and TGF- $\beta$ (Perets et al., 2003; Schmidmaier et al., 2001; Tabaka and Ikada, 1999). It was also reported that angiogenesis was enhanced when Matrigel was used together with polymer scaffolds (Laschke et al., 2008). Matrigel exists in the liquid forms at $4^{\circ} \mathrm{C}$ but forms gels at $37^{\circ} \mathrm{C}$, which allows it to easily penetrate and coat the porous polymer scaffold (Kleimann et al., 2005).

In this study, biodegradable elastic hydrogels consisting of hydrophilic PEG and hydrophobic PCL blocks were evaluated as a tissue engineering scaffold for angiogenesis. We hypothesized that the biodegradable hydrogel scaffold is suitable for supporting and growing hBMSCs, controlling the release of VEGF, and finally effectively inducing angiogenesis.

\section{MATERIALS AND METHODS}

\section{Materials}

Polycaprolactone diol (PCL diol, $\mathrm{Mn}=1250$ or 2000), benzene (anhydrous grade), acryloyl chloride, triethylamine, dimethyl sulfoxide (DMSO, anhydrous grade), and PEG diacrylate (PEG-DA, $M n=700$ ) were purchased from Sigma-Aldrich. 2,2'azobisisobutyronitrile (AIBN) was obtained from JUNSEI Chemicals (Japan) and used after purification by recrystallization in methanol. Sodium chloride powder (size distribution: 180-400 $\mu \mathrm{m}$, $99 \%$ ) was purchased from Samchun Pure Chemicals. The other chemicals were of reagent grade, and were used as received.

\section{Preparation of PEG/PCL hydrogel scaffolds}

The hydrogel scaffolds were prepared according to the same method as that previously reported (Park et al., 2007). The predetermined amounts of diacrylated PCL (PCL-DA) and PEG-DA were dissolved in $5 \mathrm{ml}$ of DMSO and placed into polypropylene conical tubes containing $7 \mathrm{~g}$ of sodium chloride salt particulates (size distribution: 180-400 $\mu \mathrm{m}, 99 \%$ ). The feed ratio between PCL-DA and PEG-DA was varied from 14:6 to $6: 14$ by weight, but the total polymer concentration was fixed at $20 \mathrm{wt} . \%$.
After radical crosslinking reaction with a small amount of AIBN at $70^{\circ} \mathrm{C}$ for $12 \mathrm{~h}$, the resultant hydrogel was cut into discs with thicknesses of $3 \mathrm{~mm}$ and immersed in distilled water and then ethyl alcohol to remove residual salt and chemicals. Finally, the scaffold was washed with distilled water several times and freeze-dried for 2-3 days.

\section{Isolation and culture of hBMSCs}

The hBMSCs were isolated and cultured as described in previous works (Bruder et al., 1997; Heo et al., 2009). The hBMSCs were maintained in an IMDM with $10 \%$ FBS, L-glutamine, penicillin $(100$ units $/ \mathrm{ml})$, and streptomycin $(100 \mu \mathrm{g} / \mathrm{ml})$ at $37^{\circ} \mathrm{C}$, in a $95 \% \mathrm{O} 2 / 5 \% \mathrm{CO}_{2}$ incubator. The hBMSCs were characterized using a mesenchymal stem cell kit (Chemicon, USA).

\section{Assessment of cell viability (MTT assay)}

Cell viability was determined by 3-(4,5-dimethylthiazol-2-yl)-2,5diphenyl tetrazolium bromide (MTT) assay (Sigma, USA). hBMSCs were seeded in 96 -well plates at $5 \times 10^{3}$ cells/well and incubated for both $48 \mathrm{~h}$ and 8 days. The MTT assay was performed according to the manufacturer's instructions. DMSO (Sigma, USA) was used to dissolve the formazan crystals for $2 \mathrm{~h}$ at room temperature, and optical density was measured at $570 \mathrm{~nm}$ using a microplate reader.

\section{Assay of VEGF secretion}

The $0.1 \mathrm{pg}$ of VEGF $(10 \mu \mathrm{g} / \mathrm{ml}, 10 \mu \mathrm{l})$ was applied on dried porous PEG:PCL(6:14) pieces and placed at the center of a 48well plate. $100 \mu$ l of VEGF reduced Matrigel was applied at the top of the scaffolds carefully allowing the scaffolds were completely coated. The scaffolds were incubated in a $37^{\circ} \mathrm{C}$ incubator for $20 \mathrm{~min}$ for complete gelation of Matrigel. $600 \mu \mathrm{l}$ of phosphate buffer solution (PBS) was added, of which $50 \mu$ of it was removed at $30,60,90,120,150,180$, and $210 \mathrm{~min}$ to determine the VEGF release. The determination of VEGF concentration was carried out with VEGF detection ELISA kit (R\&D systems).

Implantation of hydrogel scaffolds in BALB/c mice Scaffolds with a diameter of $3 \mathrm{~mm}$ (with and without hBMSC, with and without Matrigel coating) were surgically transplanted under the skin of the lateral groins of 6 week old BALB/c nude mice. After 1 to 4 weeks, the mice were sacrificed in $\mathrm{CO}_{2}$ chamber and the transplants were excised and subjected to immunohistochemistry.

Immunohistochemistry

Samples were fixed in $10 \%$ formaldehyde and paraffinized. The samples were cut into $5 \mathrm{~mm}$ of thickness and then incubated at $60^{\circ} \mathrm{C}$ for $1 \mathrm{~h}$. The slides were deparaffinized and used antigen retrieval technique for promoting sensitivity of tissue staining.

The sections were washed and then incubated in 3\% hydrogen peroxide for $6 \mathrm{~min}$. The sections were blocked for $1 \mathrm{~h}$ and stained with primary anti-CD31 antibody (1:20 dilution, rabbit polyclonal, AbCam, UK) for $30 \mathrm{~min}$, and then washed three times with Tris buffered saline (TBST) with Triton X-100. The sections were incubated in secondary HRP-conjugated anti-rabbit antibody as per the manufacturer's recommendation. After washing, the sections were counterstained with hematoxylin and then mounted with permount solution.

\section{RESULTS}

Preparation of PEG/PCL hydrogel scaffolds

Three types of porous hydrogel scaffolds with different block 

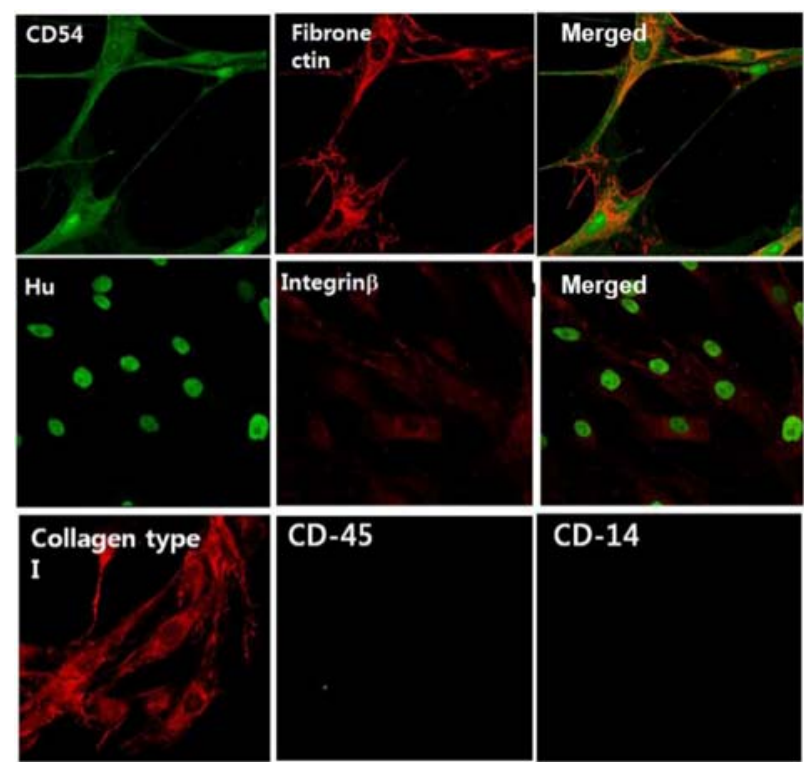

Fig. 1. Characterization of mesenchymal stem cells from human bone marrow. Cells were stained for specific mesenchymal stem cell markers with corresponding antibodies and analyzed using a confocal microscope $(\times 600)$. Positive markers: Integrin $B$, collagen type I, fibronectin, CD-54. Negative markers: CD-45, CD-14. Culture media: IMDM/20\% FBS, 2 mM Glutamine (passage 3). The right figures of two upper lanes are merge of left two figures.

compositions (PEG:PCL $=6: 14$ or $14: 6$ by weight, PCL block (MW $=1250$ or 2000) were fabricated using the salt leaching method. The polymer composition could be easily modulated by varying the feed ratio of the PEG and PCL block components to demonstrate different hydrophilicity. By using the salt leaching process, a highly porous structure could be introduced in the hydrogel samples. The characterization for chemical and physical properties of the hydrogels was performed and described in detail in our previous report (Im et al., 2007; Park et al., 2007).

\section{Determination of the identity of hBMSCs}

To determine the identity of human bone-marrow derived MSCs, we performed immunofluorescence staining for their specific markers such as collagen type I, fibronectin, CD54, Integrin $1 \beta$, and $\mathrm{Hu}$ (Fig. 1). We observed that the isolated stem cells stained positively in the cytoplasm and the nucleus for the presence of MSC marker proteins they did not show the presence of CD14, a representative marker for hematopoietic stem cells; thus, we were able to confirm that the hBMSCs were not contaminated with hematopoietic stem cells (HSCs) (Fig. 1).

Cytotoxicity of the scaffold in MSCs and growth tests To determine which type of scaffold is suitable for development of MSCs, we cultured MSCs on three different hydrogel scaffolds, and performed the assessment of cell death by MTT assay with $3 \times 10^{5}$ of the initial cells and at two different cell culture times of 2 days and 8 days. The MSCs grew on all scaffolds, and the highest growth rate was observed for the hydrogel scaffold of PEG/PCL $=6 / 14$ and PCL block of MW $=1250$ (Fig. 2). Therefore all the subsequent experiments were thus
A

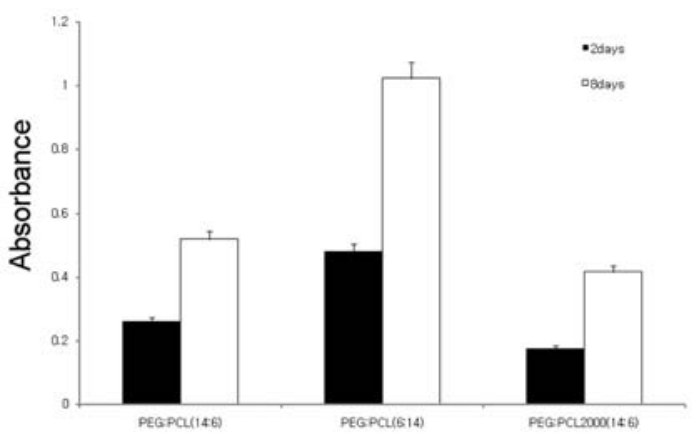

B
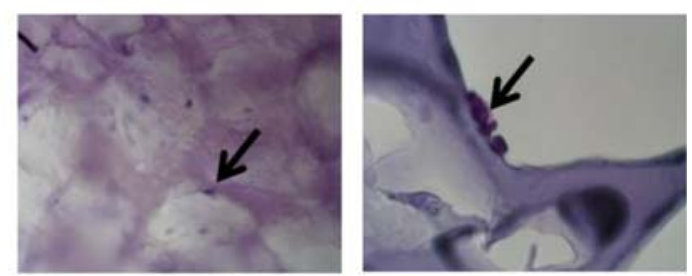

Fig. 2. Viability of hBMSC on the plate type PEG:PCL scaffolds. (A) Cells were seeded on three kinds of PEG:PCL plate type scaffolds and incubated for 2 days or 8 days. Cell viability was determined by MTT assay. (B) Low (left) and high (right) magnification of hBMSCs on PEG:PCL (6:14) scaffolds after 2 days of culture were examined using $\mathrm{H} \& \mathrm{E}$ staining.

performed with the hydrogel scaffold.

Morphological studies on biodegradable hydrogel scaffold To assess the biodegradability of the porous polymer scaffolds, we studied morphological aspects both in vitro (Figs. $3 \mathrm{~A}$ and $3 \mathrm{~B}$ ) and in vivo (Figs. $3 \mathrm{C}-3 \mathrm{E})$. It was clearly observed that the biodegradation proceeded in significantly different patterns between in vitro and in vivo. The scanning electron microscope (SEM) images from the hydrogel samples with degradation time of 2 days and 10 weeks in vitro did not show any significant morphological change. On the other hand, in in vivo, the shape of the angular parts of the pores and the corners of the material at 4 weeks (Fig. 3E) was significantly different from the shape at 2 weeks (Fig. 3D). When the hydrogel scaffold was transplanted in the BALB/c mouse to determine the biodegradability, more than $50 \%$ of the scaffold material degraded after 4 weeks (Fig. 3E).

Regulation of VEGF secretion using Matrigel coating on scaffold

Porous scaffolds may be useful for drug loading and delivery for tissue engineering, especially peptide hormones. However, the peptide hormone quickly diffuses out through their porous structure, making it difficult to achieve the desired function. Recently, various efforts have been made to control the release of growth hormones in scaffolds transplanted with cells by introducing functional groups to the biodegradable polymers used for cell transplantation (Ho et al., 2009). Here, we aimed to control the release of VEGF by coating the scaffold containing VEGF with Matrigel. The release of VEGF from the porous scaffold was saturated within $30 \mathrm{~min}$ and did not change thereafter. When the scaffold was coated with Matrigel, the VEGF 
A

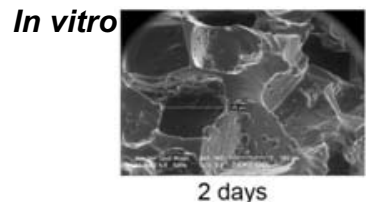

C

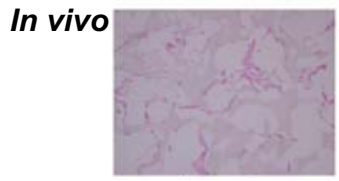

1 weeks

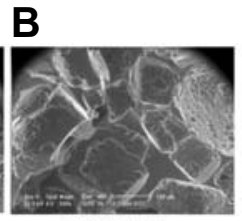

D

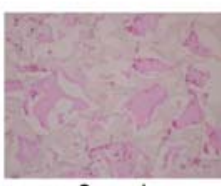

2 weeks
E

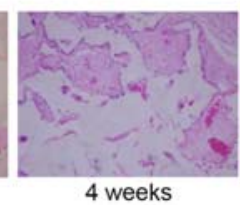

Fig. 3. Biodegradation rates of porous $P E G: P C L$ polymer in vitro and in vivo. (A, B) After incubating in IMDM with $10 \%$ FBS for 2 days and 10 weeks, scaffolds were analyzed using SEM $(\times 500)$. (B-E) Scaffolds were transplanted into the back flank of BALB/C nude mice subcutaneously for 1, 2, 4 weeks. Removed scaffolds were examined under a light microscope $(\times 400)$.

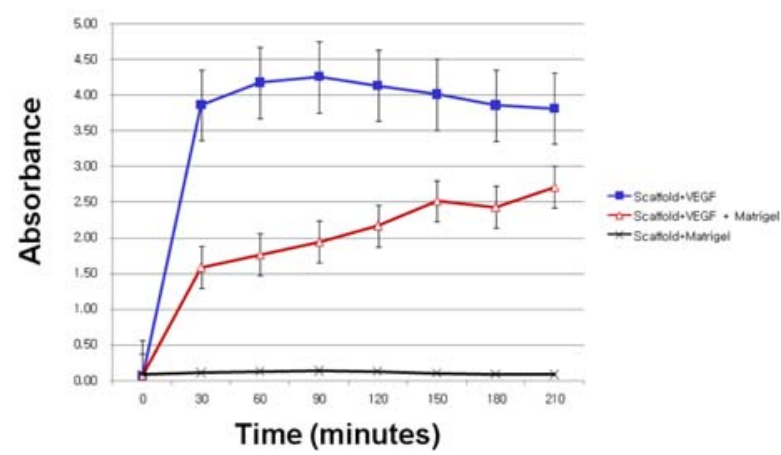

Fig. 4. Influence of Matrigel coating on the release of VEGF from porous PEG:PCL(6:14) scaffolds. Porous scaffolds scaffolds containing VEGF was coated with Matrigel were placed in PBS. Released VEGF was assayed by ELISA.

was able to be released in a controlled way for more than 210 min (Fig. 4). It indicated that the introduction of Matrigel coating on the hydrogel scaffold may allow the controlled release and prolonged functionality of VEGF.

\section{Angiogenesis by regulating VEGF release}

In order to assess the angiogenic effects of VEGF and Matrigel coating, various hydrogel scaffolds prepared with and without VEGF loading and Matrigel coating were subcutaneously implanted in BALB/c mice and immunohistochemistry for the presence of CD31 was performed after 1 week or 4 weeks. One week after implantation, the CD31 staining patterns were observed to be similar between the Matrigel coated samples and the Matrigel coated samples with VEGF (Fig. 5). In the case of the Matrigel coated samples with VEGF loading, infiltration by unknown cells showed a significant increase. Four weeks after implantation, significantly more angiogenesis was observed in the Matrigel coated scaffold with VEGF loading than in the other samples. The CD31 positive cells (vasculature) were frequently present inside the scaffold (Fig. 6).
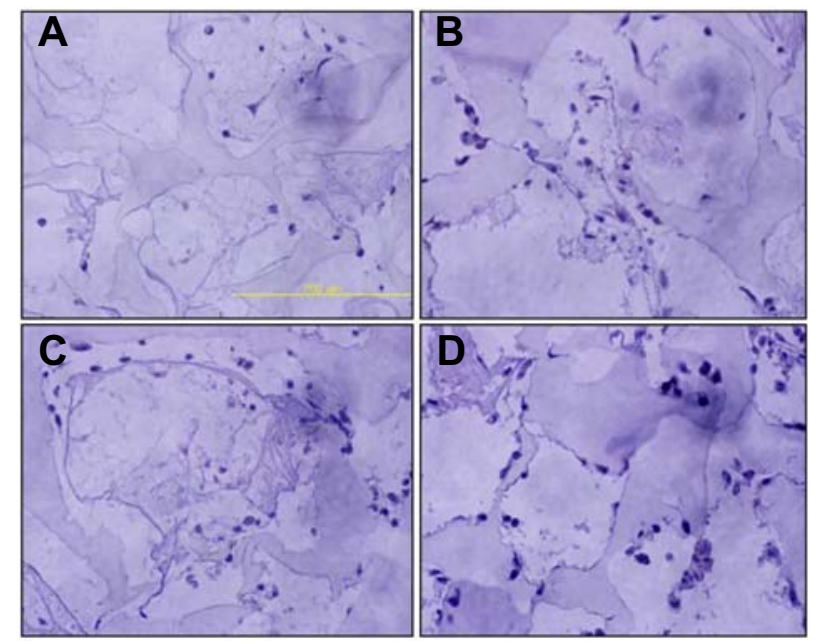

Fig. 5. Cell recruitment at early phase of transplantation by Matrigelcoated scaffolds containing VEGF. Combinatorial scaffolds were implanted into the back flank of BLAB/c nude mice subcutaneously. Immunohistochemistry analysis against CD-31 was performed after 1 week. (A) Scaffolds only, (B) Matrigel coated scaffolds, (C) Scaffolds with VEGF, and (D) VEGF containing scaffolds coated by Matrigel.
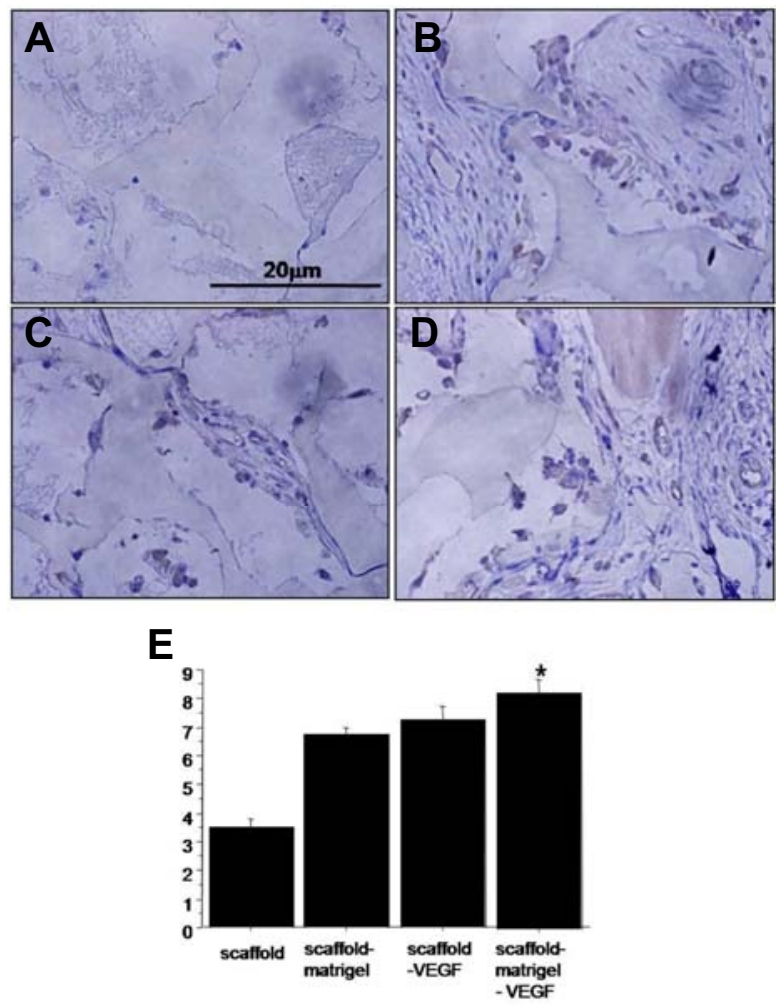

Fig. 6. Angiogenic effects of Matrigel-coated scaffolds containing VEGF. Combinatorial scaffolds were implanted into the back flank of $B L A B / c$ nude mice subcutaneously. Immunohistochemistry analysis against CD-31 was performed after 4 weeks. (A) Scaffolds only, (B) Matrigel coated scaffolds, (C) Scaffolds with VEGF, and (D) VEGF containing scaffolds coated with Matrigel. (E) Statistic analysis for CD-31 positive vessels $\left({ }^{*} p<0.05\right)$. 


\section{DISCUSSION}

This study showed that the Matrigel coated PEG/PCL hydrogel scaffold could demonstrate the controlled release of VEGF in vitro, and promoted the angiogenesis when transplanted in $\mathrm{BALB} / \mathrm{c}$ mice.

When stem cells are transplanted to treat diseases, porous polymer scaffolds are used to control stem cell growth, to maximize the effect in the treated area, and minimize excessive growth of the transplanted cells in other areas or to control differentiation (Kim et al., 2010; Lee et al., 2012). The most popular polymer scaffold materials are poly(glycolic acid) (PGA), PEG, PCL, and their copolymers (Chen et al., 2006; da Silva et al., 2011; Duailibi et al., 2004; Li et al., 2009; Schantz et al., 2003). The biodegradability of these materials depends on the microenvironment of the transplantation, and should be evaluated case by case depending on the treatment method. Previous study have reported that the PEG/PCL hydrogels did not show significant degradation in water up to 50 days; further, when the PEG content was increased to PEG:PCL= 4:1, degradation was observed to begin from 3 months and to accelerate after 5 months (Park et al., 2007). The biocompatibility of these scaffolds also depends on the type of cells to be transplanted and the combination of materials. It has been shown in our previous study that the hydrogel scaffold with PEG:PCL=14:6 resulted in the best cell growth for chondrocytes (Park et al., 2007).

The physical properties of cell transplantation polymer scaffolds, such as hardness and strength, are important factors to be considered depending on the purpose of treatment. For example, for the healing of the femur, which supports the body weight, the cutting plane receives a high stress, the healing time is long, and the material should not degrade for 8-12 weeks. Therefore, metal is therefore often used, and during regeneration, the cut surface movement is suppressed and traditional surgery is used. Cell transplantation material for this purpose has not been commercialized yet. In contrast, for cranial injuries, where the body weight is not applied, biodegradable polymer scaffolds can be used, which can biodegrade before bone regeneration (Mankani et al., 2001; Tsuchida et al., 2003). Our in vivo results showed that the PEG/PCL hydrogel scaffold degraded by more than $50 \%$ after 4 weeks. When the scaffold was implanted in the body after 1 week, more than 5 cells were observed per $150 \mu \mathrm{m}$ pore diameter, and the biodegradability was probably accelerated by these cells.

The matrigel is the most commonly used material in determining angiogenesis by physiologically active substances. Matrigel is not synthesized, but is purified from the basement membrane of an Engelbreth-Holm-Swarm tumor and contains various physiologically active substances (Kleimann et al., 2005). Both growth factor containing Matrigel (GFCM) and growth factor reduced Matrigel (GFRM) are available from the manufacturer. Matrigel exists as a liquid at a low temperature range of $0-20^{\circ} \mathrm{C}$, but becomes a gel at a temperature above $30^{\circ} \mathrm{C}$. This unique temperature-sensitive property makes it useful for introducing physiologically active substances and cells by simple mixing in a solution state and preventing migration by temperature-induced gelation. Especially, GFRM, where almost all VEGF is removed, is a great material to assess substances for angiogenesis because it does not have any angiogenic potential in itself (Kanczler et al., 2007; Lin et al., 2007; Papo et al., 2011). When stem cells adhere to polymer scaffolds for transplantation, it is difficult to engraft the stem cells in the middle of the scaffold (Kanczler et al., 2008; Lee et al., 2012; Park et al., 2010; Tanaka et al., 2009). Furthermore, timely angiogenesis is an important objective to achieve in stem cell transplantation, and many studies are being conducted to achieve this (Deng et al., 2010; Kanczler et al., 2008; Lin et al., 2007; Yang et al., 1999; 2010). When VEGF is simply applied to polymer scaffolds for angiogenesis, because of fast diffusion, the VEGF gradient disappears and appropriate angiogenesis cannot occur (Kanczler et al., 2008). Therefore, we aimed to control the release of VEGF by Matrigel coating and to maintain the VEGF concentration gradient. Our results demonstrated that the release of VEGF from the porous scaffold without Matrigel coating reached the maximum within $30 \mathrm{~min}$. On the other hand, the Matrigel coated scaffold released the VEGF in a controlled pattern for more than $210 \mathrm{~min}$ (Fig. 4).

\section{ACKNOWLEDGMENTS}

This study was financially supported by a research fund from the Chungnam National University in 2009.

\section{REFERENCES}

Bruder, S.P., Jaiswal, N., Haynesworth, S.E. (1997). Growth kinetics, self-renewal, and the osteogenic potential of purified human mesenchymal stem cells during extensive subcultivation and following cryopreservation. J. Cell. Biochem 64, 278-294.

Chen, D.C., Avansino, J.R., Agopian, V.G., Hoagland, V.D., Woolman, J.D., Pan, S., Ratner, B.D., and Stelzner, M. (2006) Comparison of polyester scaffolds for bioengineered intestinal mucosa. Cells Tissues Organs 184, 154-165.

Chen, Q., Zhang, Z., Liu, J., He, Q., Zhou, Y., Shao, G., Sun, X., Cao, X., Gong, A., and Jiang P. (2015). A fibrin matrix promotes the differentiation of EMSCs isolated from nasal respiratory mucosa to myelinating phenotypical Schwann-like cells. Mol. Cells $38,221-228$

Costa-Pinto, A.R., Correlo, V.M., Sol, P.C., Bhattacharya, M., Charbord, P., Delorme, B., Reis, R.L., and Neves, N.M. (2009). Osteogenic differentiation of human bone marrow mesenchymal stem cells seeded on melt based chitosan scaffolds for bone tissue engineering applications. Biomacromolecules 10, $2067-$ 2073.

da Silva, G.R., Junior Ada, S., Saliba, J.B., Berdugo, M. Goldenberg, B.T., Naud, M.C., Ayres, E., Orefice, R.L., and Cohen, F.B. (2011). Polyurethanes as supports for human retinal pigment epithelium cell growth. Int. J. Artif. Organs 34, 198-209.

De Kok, I.J., Peter, S.J., Archambault, M., van den Bos, C. Kadiyala, S., Aukhil, I., and Cooper, L.F. (2003). Investigation of allogeneic mesenchymal stem cell-based alveolar bone formation: preliminary findings. Clin. Oral Implants Res. 14, 481-489.

Deng, C., Zhang, P., Vulesevic, B., Kuraitis, D., Li, F., Yang, A.F., Griffith, M., Ruel, M., and Suuronen, E.J. (2010). A collagenchitosan hydrogel for endothelial differentiation and angiogenesis. Tissue Eng. Part A 16, 3099-3109.

Duailibi, M.T., Duailibi, S.E., Young, C.S., Bartlett, J.D., Vacanti, J.P., and Yelick, P.C. (2004). Bioengineered teeth from cultured rat tooth bud cells. J. Dent. Res. 83, 523-528.

Green, D.W., Kwon, H.J., and Jung, H.S. (2015). Osteogenic potency of nacre on human mesenchymal stem cells. Mol. Cells 38, 267-272.

Hennink, W.E., De Jong, S.J., Bos, G.W., Veldhuis, T.F., and van Nostrum, C.F. (2004). Biodegradable dextran hydrogels crosslinked by stereocomplex formation for the controlled release of pharmaceutical proteins. Int. J. Pharm. 277, 99-104.

Heo, J.Y., Jing, K., Song, K.S., Seo, K.S., Park, J.H., Kim, J.S., Jung, Y.J., Hur, G.M., Jo, D.Y., Kweon, G.R., et al. (2009). Downregulation of APE1/Ref-1 is involved in the senescence of mesenchymal stem cells. Stem Cells 27, 1455-1462.

Ho, Y.C., Mi, F.L., Sung, H.W., and Kuo, P.L. (2009). Heparinfunctionalized chitosan-alginate scaffolds for controlled release of growth factor. Int. J. Pharm. 376, 69-75.

Im, S.J., Choi, U.M., Subramanyam, E., Huh, K.M., and Park, K (2007). Synthesis and characterization of biodegradable elastic 
hydrogels based on poly(ethylene glycol) and poly( $\varepsilon$-caprolactone) blocks. Macromol. Res. 15, 363-369.

Kanczler, J.M., Barry, J., Ginty, P., Howdle, S.M., Shakesheff, K.M. and Oreffo, R.O. (2007). Supercritical carbon dioxide generated vascular endothelial growth factor encapsulated poly(DL-lactic acid) scaffolds induce angiogenesis in vitro. Biochem. Biophys. Res. Commun. 352, 135-141.

Kanczler, J.M., Ginty, P.J., Barry, J.J., Clarke, N.M., Howdle, S.M. Shakesheff, K.M., and Oreffo, R.O. (2008). The effect of mesenchymal populations and vascular endothelial growth factor delivered from biodegradable polymer scaffolds on bone formation. Biomaterials 29, 1892-1900.

Khare, A.R., and Peppas, N.A. (1993). Release behavior of bioactive agents from pH-sensitive hydrogels. J. Biomater. Sci. Polym. Ed. 4, 275-289.

Kim, H.J., Lee, J.H., and Im, G.I. (2010). Chondrogenesis using mesenchymal stem cells and PCL scaffolds. J. Biomed. Mater. Res. A 92, 659-666.

Kleinman, H.K., and Martin, G.R. (2005). Matrigel: basement membrane matrix with biological activity. Semin. Cancer Biol. 15, 378-386.

Laschke, M.W., Rucker, M., Jensen, G., Carvalho, C., Mulhaupt, R., Gellrich, N.C., and Menger, M.D. (2008). Incorporation of growth factor containing Matrigel promotes vascularization of porous PLGA scaffolds. J. Biomed. Mater. Res. A 85, 397-407.

Lee, K.Y., and Mooney, D.J. (2001). Hydrogels for tissue engineering. Chem. Rev. 101, 1869-1879.

Lee, H.Y., Jin, G.Z., Shin, U.S., Kim, J.H., and Kim, H.W. (2012) Novel porous scaffolds of poly(lactic acid) produced by phaseseparation using room temperature ionic liquid and the assessments of biocompatibility. J. Mater. Sci. Mater. Med. 23, 1271 1279.

Li, W.J., Chiang, H., Kuo, T.F., Lee, H.S., Jiang, C.C., and Tuan, R.S. (2009). Evaluation of articular cartilage repair using biodegradable nanofibrous scaffolds in a swine model: a pilot study. J. Tissue Eng. Regen. Med. 3, 1-10.

Lin, J., Lindsey, M.L., Zhu, B., Agrawal, C.M., and Bailey, S.R (2007). Effects of surface-modified scaffolds on the growth and differentiation of mouse adipose-derived stromal cells. J. Tissue Eng. Regen. Med. 1, 211-217.

Mankani, M.H., Kuznetsov, S..A, Fowler, B., Kingman, A., and Robey, P.G. (2001). In vivo bone formation by human bone marrow stromal cells: effect of carrier particle size and shape. Biotechnol. Bioeng. 72, 96-107.

Mehdizadeh, H., Sumo, S., Bayrak, E.S., Brey, E.M., and Cinar, A (2013). Three-dimensional modeling of angiogenesis in porous biomaterial scaffolds. Biomaterials 34, 2875-2887.

Papo, N., Silverman, A.P., Lahti, J.L., and Cochran, J.R. (2011) Antagonistic VEGF variants engineered to simultaneously bind to and inhibit VEGFR2 and alphavbeta3 integrin. Proc. Natl. Acad. Sci. USA 108, 14067-14072.

Park, J.S., Woo, D.G., Sun, B.K., Chung, H.M., Im, S.J., Choi, Y.M., Park, K., Huh, K.M., and Park, K.H. (2007). In vitro and in vivo test of PEG/PCL-based hydrogel scaffold for cell delivery application. J. Control. Release 124, 51-59.

Park, J.S., Yang, H.J., Woo, D.G., Yang, H.N., Na, K., and Park K.H. (2010). Chondrogenic differentiation of mesenchymal stem cells embedded in a scaffold by long-term release of TGF-beta 3 complexed with chondroitin sulfate. J. Biomed. Mater. Res.
A 92, 806-816.

Perets, A., Baruch, Y., Weisbuch, F., Shoshany, G., Neufeld, G., and Cohen, S. (2003). Enhancing the vascularization of threedimensional porous alginate scaffolds by incorporating controlled release basic fibroblast growth factor microspheres. $J$. Biomed. Mater. Res. A 65, 489-497.

Peters, M.C., Polverini, P.J., and Mooney, D.J. (2002). Engineering vascular networks in porous polymer matrices. J. Biomed. Mater. Res. 60, 668-678.

Phinney, D.G., and Prockop, D.J. (2007). Concise review: mesenchymal stem/multipotent stromal cells: the state of transdifferentiation and modes of tissue repair--current views. Stem Cells 25, 2896-2902.

Pittenger, M.F., Mackay, A.M., Beck, S.C., Jaiswal, R.K., Douglas, R., Mosca, J.D., Moorman, M.A., Simonetti, D.W., Craig, S., and Marshak, D.R. (1999). Multilineage potential of adult human mesenchymal stem cells. Science 284, 143-147.

Schantz, J.T., Hutmacher, D.W., Lam, C.X., Brinkmann, M., Wong, K.M., Lim, T.C., Chou, N., Guldberg, R.E., and Teoh, S.H. (2003). Repair of calvarial defects with customised tissueengineered bone grafts II. Evaluation of cellular efficiency and efficacy in vivo. Tissue Eng. 9 (Supp/ 1), S127-139.

Schmidmaier, G., Wildemann, B., Stemberger, A., Haas, N.P., and Raschke, M. (2001). Biodegradable poly(D,L-lactide) coating of implants for continuous release of growth factors. J. Biomed. Mater. Res. 58, 449-455.

Tabata, Y., and Ikada, Y. (1999). Vascularization effect of basic fibroblast growth factor released from gelatin hydrogels with different biodegradabilities. Biomaterials 20, 2169-2175.

Tanaka, T., Hirose, M., Kotobuki, N., Tadokoro, M., Ohgushi, H., Fukuchi, T., Sato, J., and Seto, K. (2009). Bone augmentation by bone marrow mesenchymal stem cells cultured in threedimensional biodegradable polymer scaffolds. J. Biomed. Mater. Res. A 91, 428-435.

Tsuchida, H., Hashimoto, J., Crawford, E., Manske, P., and Lou, J. (2003). Engineered allogeneic mesenchymal stem cells repair femoral segmental defect in rats. J. Orthop. Res. 21, 44-53.

Yang, X., Castilla, L.H., Xu, X., Li, C., Gotay, J., Weinstein, M., Liu, P.P., and Deng, C.X. (1999). Angiogenesis defects and mesenchymal apoptosis in mice lacking SMAD5. Development $126,1571-1580$

Yang, X.B., Webb, D., Blaker, J., Boccaccini, A.R., Maquet, V., Cooper, C., and Oreffo, R.O. (2006). Evaluation of human bone marrow stromal cell growth on biodegradable polymer/bioglass composites. Biochem. Biophys. Res. Commun. 342, 1098-1107.

Yang, F., Cho, S.W., Son, S.M., Bogatyrev, S.R., Singh, D., Green, J.J., Mei, Y., Park, S., Bhang, S.H., Kim, B.S., et al. (2010) Genetic engineering of human stem cells for enhanced angiogenesis using biodegradable polymeric nanoparticles. Proc. Natl. Acad. Sci. USA 107, 3317-3322.

Zanatta G. Rudisile, M. Camassola, M. Wendorff, J, Nardi, N. Gottfried, C., Pranke, P., and Netto, C.A. (2012). Mesenchymal stem cell adherence on poly(D, L-lactide-co-glycolide) nanofibers scaffold is integrin-beta 1 receptor dependent. J. Biomed. Nanotechnol. 8, 211-218.

Zeiser, R., Marks, R., Bertz, H., and Finke, J. (2004). Immunopathogenesis of acute graft-versus-host disease: implications for novel preventive and therapeutic strategies. Ann. Hematol. 83, $551-565$. 